\title{
Large coherent acoustic-phonon oscillation observed in InGaN/GaN multiple-quantum wells
}

\author{
Chi-Kuang Sun a) and Jian-Chin Liang \\ Department of Electrical Engineering and Institute of Electro-Optical Engineering, \\ National Taiwan University, Taipei 10617, Taiwan, Republic of China \\ Christopher J. Stanton \\ Department of Physics, University of Florida, Gainesville, Florida 32611
}

Amber Abare, Larry Coldren, and Steven P. DenBaars

Department of Electrical and Computer Engineering, University of California, Santa Barbara, California 93106

(Received 9 March 1999; accepted for publication 2 July 1999)

\begin{abstract}
We have observed coherent acoustic-phonon oscillation in InGaN/GaN multiple-quantum wells. With femtosecond pulse excitation, photogenerated carriers screen out the strained-induced piezoelectric field and initiate the coherent acoustic-phonon oscillation. The resulted modulation of the piezoelectric field will then cause large optical property oscillation through the quantum-confined Stark effect. (C) 1999 American Institute of Physics. [S0003-6951(99)01335-2]
\end{abstract}

Progress in femtosecond lasers and ultrafast spectroscopy technology has enabled us to generate and directly observe the coherent oscillation of phonon modes. ${ }^{1-7}$ Oscillation changes in optical properties have been observed in $\mathrm{GaAs},{ }^{1,2} \mathrm{Ge},{ }^{3}$ and a number of other materials. ${ }^{4,5}$ In semiconductors, carriers can be excited by ultrashort pump pulses in a spatial area much larger than 1 lattice unit cell and the excited carrier populations (with wave vector $q \cong 0$ ) are coupled to the corresponding optical-phonon modes (also with $q \cong 0$ ). ${ }^{6}$ Besides being incoherently populated, some of these optical phonon modes can behave like classical oscillators and have nonzero time-dependent displacement. This is different from spontaneous emission of phonons, which is an incoherent process that populates the phonon modes with the expectation value of each mode being zero. The corresponding modulation of the material dielectric constant, due to coherent oscillation of optical phonons, could then be observed by changes in the intensities of transmitted or reflected probe light pulses.

For acoustic phonons, it is much harder to selectively excite a specific acoustic phonon mode by $q \cong 0$ carriers in bulk materials. With the aid of laser-induced grating techniques, coherent acoustic waves of a few $\mathrm{MHz}$ to $30 \mathrm{GHz}$ can be induced through periodic optical fields, photothermal gratings, or the photon-excited periodic carrier distribution, all with nonzero corresponding wave vectors. ${ }^{8,9}$ Similar techniques can be applied to high-frequency acoustic wave generation by using a multilayer structure ${ }^{10}$ or a repeated nanostructure ${ }^{11}$ where larger wave vectors can be achieved. With the acoustic wave reflection on the boundary of a thin fine structure, optical reflection oscillations corresponding to the round-trip time have also been previously observed ${ }^{12}$ and coherent acoustic-phonon oscillations corresponding to spherical acoustic modes in quantum dots were also

a)Electronic mail: sun@cc.ee.ntu.edu.tw reported. ${ }^{7}$ Semiconductor superlattices exhibit zone folding of the acoustic branches within the mini-Brillouin zone due to the artificial periodicity, also enabling the observation of coherent oscillation of the zone-folded acoustic phonons in AlAs/GaAs superlattices. ${ }^{13,14}$ However, the observed reflection modulation $\Delta R / R$ is extremely small, on the order of $10^{-5}$, such that a step-and-repeat detection scheme with a fast scanning time delay is required to maintain a signal-tonoise ratio of better than $10^{-6}$ in femtosecond pump-andprobe systems. ${ }^{14}$ The coherent phonon excitation is probably due to photoexcited carriers-induced stress in the well layers, ${ }^{13}$ and the detection is based on refractive index changes caused by acoustic deformation potential-exciton coupling. ${ }^{14}$

In this letter, we demonstrate that in multiple-quantum wells (MQWs) with strain-induced piezoelectric-field, strong coherent acoustic-phonon oscillation can be easily generated and the large modulated absorption in probed pulses with $\Delta T / T$ on the order of $10^{-2}$ can thus be observed. The oscillation frequency can be tuned by changing the period width of the multiple-quantum wells.

The experiments were performed at room temperature on 14 periods of InGaN/GaN MQWs. The recent demonstration of high-brightness light-emitting and laser diodes with InGaN MQWs as active regions ${ }^{15}$ has established the III-V nitrides as key materials for optoelectronics operating in the green-UV wavelength range. Because of the large piezoelectric constants along the [0001] orientation in group III nitrides, a high strain-induced piezoelectric field is expected (on the order of $\mathrm{MV} / \mathrm{cm}$ ) and would play an important role in the optical properties of (0001)-oriented InGaN quantum wells (QWs) due to the quantum-confined Stark effect (QCSE). ${ }^{16}$ Even though the critical thickness of InGaN/GaN is usually thinner than the heterojunction overlayer thickness and the dense threading defects provide strain relief mechanisms, the heterojunction overlayers are thought to be partially strained to an unknown degree. The InGaN/GaN 


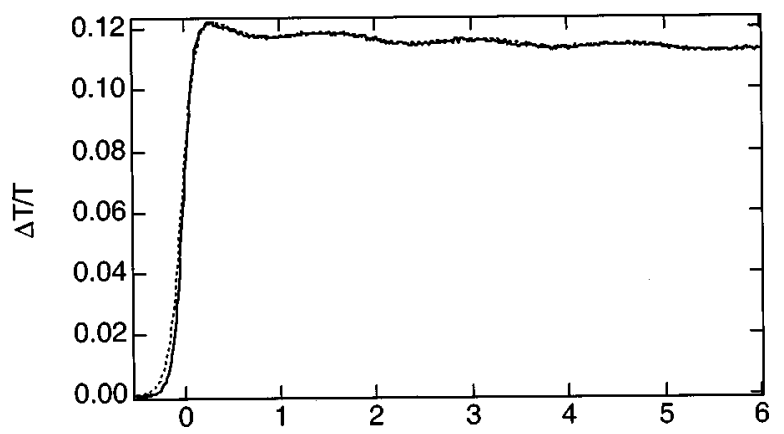

FIG. 1. Measured transient transmission changes vs probe delay for $62 / 43 \AA$ InGaN/GaN MQW. The dotted line is a convolution fit using a positive cosine oscillation with a period of $1.5 \mathrm{ps}$.

MQWs were grown on $c$-plane sapphire using atmosphericpressure metal-organic chemical-vapor deposition. After annealing the substrate at $1050^{\circ} \mathrm{C}$, a 19 -nm-thick GaN nucleation layer was deposited at $525^{\circ} \mathrm{C}$. The temperature was then raised to $1080^{\circ} \mathrm{C}$ to grow $\mathrm{GaN}$ of $2.5 \mu \mathrm{m}$ thickness. The 14 periods of InGaN/GaN MQWs were then grown on this thick GaN layer at a temperature of $790{ }^{\circ} \mathrm{C}$. After the MQW growth, a 12-nm-thick $\mathrm{Al}_{0.1} \mathrm{Ga}_{0.9} \mathrm{~N}$ was grown at low pressure. The temperature was then raised to $1060^{\circ} \mathrm{C}$ to finish a $0.1-\mu \mathrm{m}$-cap $\mathrm{Al}_{0.1} \mathrm{Ga}_{0.9} \mathrm{~N}$ layer. The barrier widths of all samples were held at $43 \AA$ while the well widths were varied from 12 to $62 \AA$. The crystal structure was wurzite. The In composition in the MQWs was determined to be around $6 \%$ by $\mathrm{x}$-ray diffraction. The room-temperature photoluminescence study and absorption measurements indicated that the band gaps of the MQWs were between 390 and $430 \mathrm{~nm}$ for 12-62 $\AA$ well-width MQWs. The room-temperature bandgap energy of bulk GaN was $365 \mathrm{~nm}$.

The experiments were performed using a standard single-color femtosecond transmission pump-probe technique with a Kerr-lens Mode-locked Ti:sapphire laser. The laser output pulses from the femtosecond mode-locked Ti:sapphire laser were frequency doubled in a BBO crystal to reach the band-gap energy of the InGaN/GaN MQWs. The frequency-doubled pulses had a pulse width of $180 \mathrm{fs}$ at a wavelength of $390 \mathrm{~nm}$ measured by a two-photon absorption-type pump-probe autocorrelation in a bulk GaN thin film. ${ }^{17}$ The femtosecond UV pump pulses photoexcite carriers in the MQWs. During the relaxation processes of electrons and holes, due to the periodic distribution of the photoexcited carrier population, carriers are easily coupled to the selective acoustic-phonon mode with wave vector $q$ corresponding to the inverse period width with $q=2 \pi / a$, where $a$ is the MQW period width. Coherent oscillation of this coupled phonon mode was impulsively induced by carrier screening of the piezoelectric field in the QW by photoexcited carriers, and the induced coherent oscillation with nonzero time-dependent displacement will thus modulate the strain-induced piezoelectric field and results in transmission changes through the QCSE. With a new equilibrium position due to screening of photoexcited carriers, a cosine-like oscillation should be expected.

Figure 1 shows the measured probe transmission change as a function of probe delay for a $62 \AA$ well-width MWQ for a pump wavelength of $394 \mathrm{~nm}$. After the pump excited carriers in the quantum wells and caused transmission increases

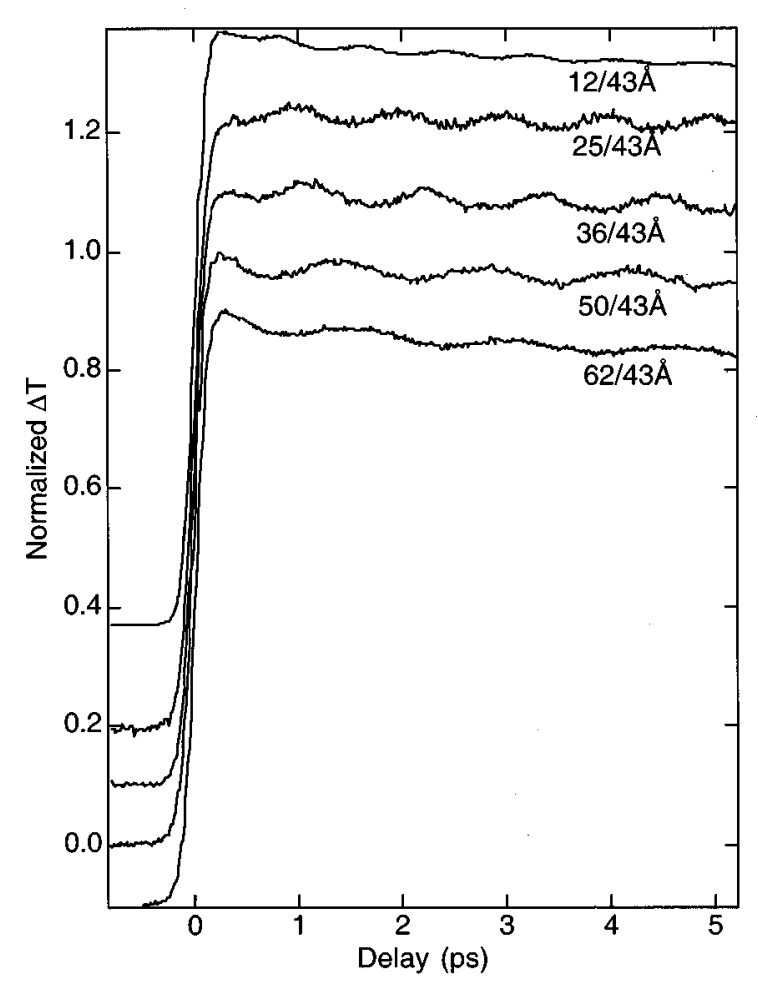

FIG. 2. Normalized transient transmission changes vs probe delay for different MQWs. Traces are vertically displaced for clarity.

at zero time delay, a clear cosine-like transmission oscillation was observed. The observed oscillation frequency was found to be independent of pump/probe photoenergy and pump fluence. We attribute this large oscillation to coherent longitudinal-acoustic (LA) phonon oscillation in the QW growth direction ( $c$ axis). The observed oscillation period was $1.5 \mathrm{ps}$, corresponding to a frequency of $0.67 \mathrm{THz}$. The phase component of the cosine argument was always close to zero, with a time delay less than the pulse width, suggesting that the excitation of the oscillation was displacive $e^{4}$ and the system was under a new equilibrium position due to carrier screening after photoexcitation. ${ }^{18}$ The corresponding pump fluence and generated carrier density were $160 \mu \mathrm{J} \mathrm{cm}^{-2}$ and $1.1 \times 10^{12} \mathrm{~cm}^{-2} /$ per-well. When the pumped carriers screened the tensile-strain (in the $c$ axis) -induced piezoelectric field and cause the opposite charged ions to oscillate away from or toward each other, the piezoelectric field will be increased or reduced with a modulated quantum-confined Stark effect. The electron-hole wave-function overlap will thus be modulated and reflected in probe transmission changes. This conforms to the observed cosine oscillation on top of the transmission change background observed in Fig. 1. For the trace of Fig. 1, the total transmission changes $\Delta T / T$ is on the order of $10^{-1}$. Large transmission modulation due to coherent acoustic-phonon oscillation on the order of $10^{-2}$ was easily observed with a generated twodimensional carrier density of $1 \times 10^{12} \mathrm{~cm}^{-2}$ per well.

With different periodic distributions of the photoexcited carrier population, carriers are coupled to the selective acoustic-phonon mode with wave vector $q$ corresponding to the inverse period width with $q=2 \pi / a$, where $a$ is the MQW period width. Figure 2 shows the normalized probe transmission changes as a function of probe delay for differ- 


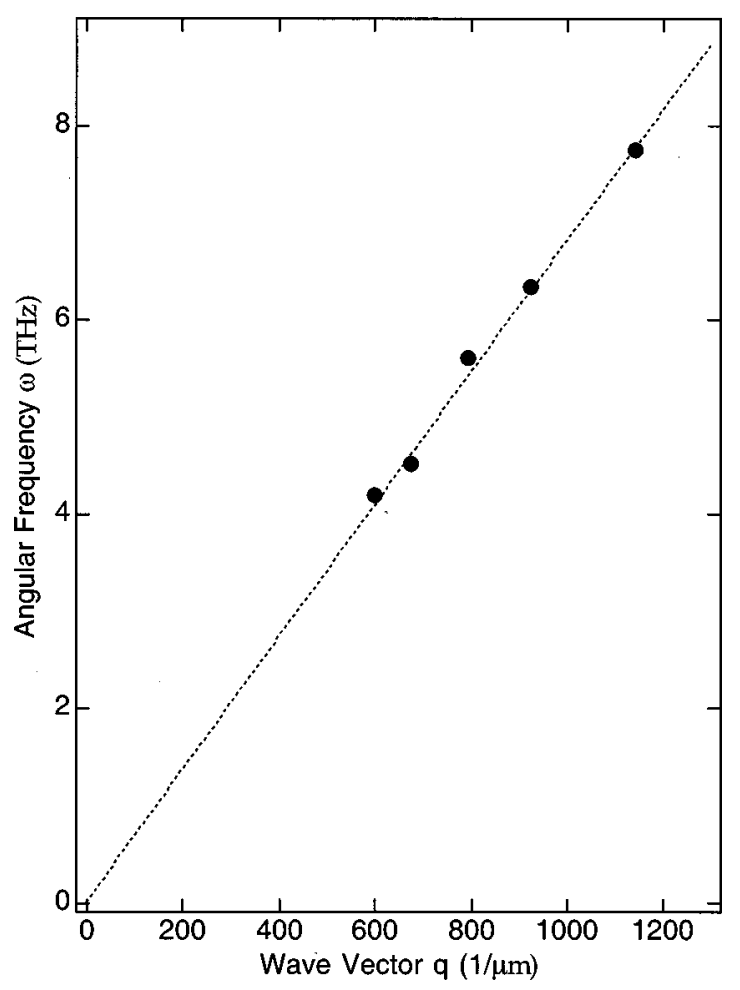

FIG. 3. Measured angular frequency of oscillation vs carrier wave vector for different MQWs. The dashed line corresponds to a sound velocity of 6800 $\mathrm{m} / \mathrm{s}$.

ent MQWs with photon energies above the quantum-well band gap. The oscillation frequency varies from $1.23 \mathrm{THz}$ for a QW period of $55 \AA$ (12 $⿱$ A well) to $0.67 \mathrm{THz}$ for a QW period of $105 \AA$ ( $62 \AA$ well). Figure 3 plots the observed acoustic-phonon angular frequency versus the excited phonon wave vector, which is a function of period width. A linear relation is observed. The slope of the linear relation corresponds to a sound velocity of $6800 \mathrm{~m} / \mathrm{s}$ for the LA mode in the [0001] direction in wurzite GaN. Our measured value agrees well with the previously obtained LA mode $c$-axis sound velocity of $6620 \pm 220 \mathrm{~m} / \mathrm{s}$, which is derived from the results of an elastic stiffness constant $c_{33}$ computation at room temperature using the mean-square displacement of GaN measured by an x-ray method, ${ }^{19}$ assuming a density of $6.095 \mathrm{~g} / \mathrm{cm}^{3}$. This supports our hypothesis that the observed oscillation is due to coherent acoustic-phonon oscillation in the GaN MQW.

In summary, we have observed coherent acousticphonon oscillation in InGaN/GaN multiple-quantum wells.
This oscillation is induced by the coupling between the periodic carrier population and the corresponding acousticphonon mode. After femtosecond pulse excitation, photogenerated carriers will screen out the strain-induced piezoelectric field and initiate this coherent acoustic-phonon oscillation. The resulting modulation of the piezoelectric field will then cause large optical property oscillation through the quantum-confined Stark effect. Transmission changes on the order of $10^{-2}$ can thus be easily observed. Our work opens the way to the studies of the properties of acoustic phonons and their interactions with surrounding carriers in semiconductors.

The authors would like to acknowledge stimulating scientific discussions with Professor E. P. Ippen, Professor D. H. Reitze, and Professor Y.-M. Chang. This work is supported by the National Science Council of R.O.C (Taiwan) under Grant No. NSC 88-2112-M-002-003. C. J. S. acknowledges support from NSF through Grant No. DMR 9817828.

${ }^{1}$ G. C. Cho, W. Kutt, and H. Kurz, Phys. Rev. Lett. 65, 764 (1990).

${ }^{2}$ W. Kutt, G. C. Cho, T. Pfeifer, and H. Kurz, Semicond. Sci. Technol. 7, B77 (1992).

${ }^{3}$ T. Pfeifer, W. Kutt, H. Kurz, and H. Scholz, Phys. Rev. Lett. 69, 3248 (1992).

${ }^{4}$ T. K. Cheng, J. Vidal, H. J. Zeiger, G. Dresselhaus, M. S. Dresselhaus, and E. P. Ippen, Appl. Phys. Lett. 59, 1923 (1991).

${ }^{5}$ W. Albrecht, T. Kruse, and H. Kurz, Phys. Rev. Lett. 69, 1451 (1992).

${ }^{6}$ A. V. Kuznetsov and C. J. Stanton, Phys. Rev. B 51, 7555 (1995).

${ }^{7}$ E. R. Thoen, G. Steinmeyer, P. Langlois, E. P. Ippen, G. E. Tudury, C. H. Brito Cruz, L. C. Barbosa, and C. L. Sedar, Appl. Phys. Lett. 73, 2149 (1998).

${ }^{8}$ A. Harata, H. Nishimura, and T. Sawada, Appl. Phys. Lett. 57, 132 (1990).

${ }^{9}$ M. D. Fayer, IEEE J. Quantum Electron. 22, 1437 (1986).

${ }^{10}$ P. Basséras, S. M. Gracewski, G. W. Wicks, and R. J. D. Miller, J. Appl. Phys. 75, 2761 (1994).

${ }^{11}$ N.-H. Lin, H. J. Maris, L. B. Freund, K. Y. Lee, H. Luhn, and D. P. Kern, J. Appl. Phys. 73, 37 (1993).

${ }^{12}$ H. T. Grahn, D. A. Young, H. J. Maris, J. Tauc, J. M. Hong, and T. P. Smith III, Appl. Phys. Lett. 53, 2023 (1988).

${ }^{13}$ A. Yamamoto, T. Mishina, and Y. Masumoto, Phys. Rev. Lett. 73, 740 (1994).

${ }^{14}$ A. Bartels, T. Dekorsy, H. Kurz, and K. Köhler, Phys. Rev. Lett. 82, 1044 (1999).

${ }^{15}$ S. Nakamura and G. Fasol, The Blue Laser Diode (Springer, Berlin, 1997).

${ }^{16}$ T. Takeuchi, S. Sota, M. Katsuragawa, M. Komori, H. Takeuchi, H. Amano, and I. Akasaki, Jpn. J. Appl. Phys., Part 2 36, L382 (1997).

${ }^{17}$ C.-K. Sun, Y.-L. Huang, J.-C. Wang, S. Keller, M. Mack, U. K. Mishra, and S. P. DenBaars, in Technical Digest of the Conference on Laser and Electro-optics, Baltimore, MD, Paper No. CTuI4 (1999).

${ }^{18}$ L. H. Peng, C. W. Chung, and L.-H. Lou, Appl. Phys. Lett. 74, 795 (1999).

${ }^{19}$ V. A. Savastenko and A. U. Sheleg, Phys. Status Solidi A 48, K135 (1978). 